\title{
Genetic variation of native Perilla germplasms collected from South Korea using SSR markers and morphological characteristics
}

\author{
Jun Seok Oh ${ }^{1,2, \dagger}$, Kyu Jin Sa ${ }^{1, \dagger}$, Hyeon Park ${ }^{1,2, \dagger}$, Do Yoon Hyun ${ }^{3}$, Sookyeong Lee ${ }^{3}$, Ju Hee \\ Rhee $^{3}$, and Ju Kyong Lee ${ }^{1,2 *}$
}

\author{
${ }^{1}$ Department of Applied Plant Sciences, College of Agriculture and Life Sciences, Kangwon National \\ University, Chuncheon 24341, Korea \\ ${ }^{2}$ Interdisciplinary Program in Smart Agriculture, Kangwon National University, Chuncheon 24341, Korea \\ ${ }^{3}$ National Agrobiodiversity Center, National Institute of Agricultural Sciences, RDA, Jeonju 54874, Korea \\ *Correspondence: jukyonglee@kangwon.ac.kr; Tel.: +82-33-250-6415, Fax: +82-33-255-5558 \\ ${ }^{\dagger}$ These authors contributed equally to this work.
}

\begin{abstract}
Using morphological characteristics and SSR markers, we evaluated the morphological and genetic variation of 200 Perilla accessions collected from the five regions of South Korea and other region. In morphological characteristics analysis, particularly, leaf color, stem color, degree of pubescence, leaf size were found to be useful for distinguishing the characteristics of native Perilla accessions cultivated in South Korea. A total of 137 alleles were identified in the 20 simple sequence repeat (SSR) markers, and the number of alleles per locus ranged from 3 to 13, and the average number of alleles per locus was 6.85. The average gene diversity (GD) was 0.649, with a range of 0.290-0.828. From analysis of SSR markers, accessions from the Jeolla-do and Gyeongsang-do regions showed comparatively high genetic diversity values compared with those from other regions in South Korea. In the unweighted pair group method with arithmetic mean (UPGMA) analysis, the 200 Perilla accessions were found to cluster into three major groups and an outgroup with a genetic similarity of $42 \%$, and did not showed a clear geographic structure from the five regions of South Korea. Therefore, it is believed that landrace Perilla seeds are frequently exchanged by farmers through various routes between the five regions of South Korea. The results of this study are expected to provide useful information for conservation of these genetic resources and selection of useful resources for the development of varieties for seeds and leafy vegetables of cultivated var. frutescens of Perilla crop in South Korea.
\end{abstract}

Keywords: Perilla crop; genetic resources; morphological traits; principal component analysis; SSR marker; genetic variation

\section{Introduction}

Perilla frutescens (L.) Britt. is an annual self-fertilizing crop of Lamiaceae. Perilla crop has been traditionally cultivated and used, mainly in Asian countries, especially in East Asia, and is divided into two different varieties (or cultivated types), P. frutescens var. frutescens and var. crispa, based on their distinct morphological characteristics [1-4]. For example, Perilla frutescens var. frutescens is used as an oil and leafy vegetable crop, and $P$. frutescens var. crispa is used as a vegetable and herbal medicine crop [5-7]. These two varieties of Perilla crop have a long history of cultivation in East Asia [2, 6, 7, 8]. Although Perilla species is widely distributed and used in the Himalayan hills, East Asia, and Southeast Asia, Perilla crop is considered to originate in East Asia because many genetic resources of two varieties of Perilla crop and their weedy types are distributed and used in the region as a traditional folk crop (oil crop, Chinese medicine crop, or vegetable crop) [2-7]. Furthermore, the two varieties of Perilla crop are different for their morphological characters. Cultivated P. frutescens var. frutescens has green leaves and stem, and has non-wrinkled leaves (Supplement Fig. 1a). In contrast, cultivated $P$. frutescens var. crispa has red or green leaves, has wrinkly or non-wrinkly leaves (Supplement Fig. 1b)

Today, in East Asia, the two cultivated types of Perilla crop are widely cultivated and used in South Korea and Japan $[3,5,6,9]$. Namely, the cultivated type of $P$. frutescens var. frutescens is widely cultivated in South Korea as both an oil crop and a vegetable crop. The leaves of cultivated P. frutescens var. frutescens are preferred as salad vegetables that are eaten with meat and used for pickles in South Korea. Furthermore, Perilla seed oil of cultivated $P$. frutescens var. frutescens has a high content of linolenic acid, which is a polyunsaturated fatty 
acid such as linoleic acid (18:2) and $\alpha$-linolenic acid (18:3), comprise approximately $80 \%$ of Perilla seed oil [10-13]. Additionally, the seeds are used like sesame seeds for flavoring agents or seasoning in Korean traditional foods. Therefore, the cultivation area of cultivated $P$. frutescens var. frutescens has increased greatly, and its leaves and seed oil have been in the limelight as health foods in South Korea [9, 13, 14]. On the other hand, the cultivated $P$. frutescens var. crispa is widely cultivated in Japan and used for leafy vegetable or pickles $[4,6]$.

The conservation of genetic resources is primarily aimed at ensuring the proper preservation and storage of genetic resources belonging to economically important crops that have been collected in genebanks [14-16]. In addition, for the successful use and selection of genetic resources that can be used for crop improvement, it is most important to evaluate and understand the genetic diversity of genetic resources preserved in genebanks [15, 16]. In Perilla crop, many researchers have been used to analyze genetic diversity, genetic relationships, and population structure of accessions of cultivated and weedy types of Perilla crop using several DNA molecular marker systems including random amplification of polymorphic DNAs (RAPD) [17], amplified fragment length polymorphisms (AFLP) [3], and simple sequence repeats (SSRs) [9, 13, 14, 18, 19; 20]. Among these DNA molecular markers, particularly SSR markers have provided useful information for the analysis of genetic diversity, genetic relationships, population structure, and association mapping in the germplasm of Perilla crop because they are highly polymorphic, generally codominant, reproducible, and abundant in plant genomes [19-23]. Therefore, SSR markers are expected to be useful for analysis of the genetic diversity, genetic relationships, and population structure of the germplasm of cultivated $P$. frutescens var. frutescens of Perilla crop.

Furthermore, to ensure the long-term success of breeding programs and to make the best use of the germplasm resources of Perilla crop preserved in the RDA-Genebank of the Republic of Korea (http://genebank.rda.go.kr), it is necessary to understand the morphological variations among accessions of cultivated $P$. frutescens var. frutescens in South Korea. In particular, morphological variations in cultivated crop species and their cultivars or native varieties can be affected by the growing environment, geographic distribution, and cultivation history within their cultivated area or habitat [24-27]. Thus, it is necessary to clarify the geographical patterns of morphological variations of germplasm resources of cultivated $P$. frutescens var. frutescens in South Korea for the efficient management and selection of these germplasms.

Therefore, in this study, we used morphological characteristics and SSR markers to evaluate the morphological variation, genetic diversity, genetic relationships, and population structure of germplasm resources of cultivated $P$. frutescens var. frutescens of Perilla crop collected in South Korea.

\section{Results}

2.1. Morphological variation in the accessions of cultivated var. frutescens of Perilla crop

The results of three quantitative and eight qualitative traits for 183 accessions of cultivated $P$. frutescens var. frutescens are summarized in Supplement Table 1.

In the survey of eight qualitative traits, for color of leaf surface (QL1), 22 accessions showed light green color, 100 accessions showed green color, and 61 accessions showed dark green color. For color of reverse side leaf (QL2), 65 accessions showed light green, 103 accessions showed green, and 15 accessions showed dark green. For stem color (QL3), 22 accessions showed light green, 100 accessions showed green, and 61 accessions showed dark green. For seed color (QL4), 17 accessions showed white, 8 accessions showed gray, 115 accessions showed brown, and 43 accessions showed dark brown. For leaf shape (QL5), 97 accessions showed lanceolate shape, 70 accessions showed heart shape, and 16 accessions showed oval shape. For degree of pubescence (QL6), 72 accessions showed slightly pubescent, 108 accessions showed normal pubescent, and 3 accessions showed heavily pubescent. For seed hardness (QL7), 174 accessions had soft seeds and 9 accessions had hard seeds. In the case of plant fragrance (QL8), 167 accessions showed the plant fragrance of cultivated P. frutescens var. frutescens and 16 accessions showed a slightly different plant fragrance (Supplement Table 1).

In the survey of three quantitative traits, leaf width (QN1) of the 183 accessions was distributed as follows: 50 accessions were less than $8.0 \mathrm{~cm}, 69$ accessions were $8.1 \sim 9.0 \mathrm{~cm}, 43$ accessions were $9.1 \sim 10.0 \mathrm{~cm}, 13$ accessions were $10.1 \sim 11.0 \mathrm{~cm}, 6$ accessions were $11.1 \sim 12.0 \mathrm{~cm}$, and 6 accessions were $12.1 \sim 13.0 \mathrm{~cm}$. For leaf length (QN2), the 183 accessions were distributed as follows: 6 accessions were less than $10.0 \mathrm{~cm}, 23$ accessions were $10.1 \sim 11.0 \mathrm{~cm}, 28$ accessions were $11.1 \sim 12.0 \mathrm{~cm}, 53$ accessions were $12.1 \sim 13.0 \mathrm{~cm}, 42$ accessions were $13.1 \sim 14.0 \mathrm{~cm}, 19$ accessions were $14.1 \sim 15.0 \mathrm{~cm}$, and 12 accessions were more than $15.1 \mathrm{~cm}$. For flowering time (QN3), the 183 accessions were divided into two types as follows: 104 accessions showed intermediate maturing type (flowering days from August 15 to September 5) and 79 accessions showed late maturing type (flowering days after September 6). We did not find any early maturing type among the 183 accessions used in this study (Supplement Table 1). 
2.2. Principal component analysis (PCA) among 183 accessions of cultivated P. frutescens var. frutescens of Perilla crop

The first and second principal components accounted for $20.4 \%$ and $14.5 \%$, respectively, of the total variance (Table 1). In the PCA analysis, QL1, QL3, QN1, QN2, QL5, QL4, and QL7 contributed in the positive direction on the first axis, and QL2, QL6, OL8, and QN3 contributed in the negative direction on the first axis. Among the 11 quantitative and qualitative traits, QL1, QL2, QL3, QL6, OL8, QN1, and QN2 contributed relatively significantly in the positive or negative direction on the first axis, while QL1, QL3, QN1, QN2, and QN3 contributed relatively significantly in the positive or negative direction on the second axis. Thus, these traits that contributed in the positive or negative direction on the first or second axis (Table 1) are considered to be useful for discrimination between accessions of cultivated P. frutescens var. frutescens. In particular, along axis 1 of the PCA analysis (Fig. 1), most accessions of cultivated P. frutescens var. frutescens were clearly separated, that is, all accessions of cultivated $P$. frutescens var. frutescens formed three groups on the positive and negative sides of the first axis (Fig. 1).

Table 1. Cumulative variance of first and second principal components and the loadings of 11 qualitative and quantitative characters on each principal component.

\begin{tabular}{lcc}
\hline \multirow{2}{*}{ Morphological Character } & \multicolumn{2}{c}{ Eigenvectors } \\
\cline { 2 - 3 } & 1 & 2 \\
\hline QL2 (Color of reverse side leaf) & -0.211 & -0.040 \\
QL6 (Degree of pubescence) & -0.096 & -0.304 \\
QL8 (Plant fragrance) & -0.062 & -0.061 \\
QN3 (Flowering time) & -0.009 & -0.534 \\
QL7 (Seed hardness) & 0.120 & 0.108 \\
QL4 (Seed color) & 0.146 & -0.241 \\
QL5 (Leaf shape) & 0.197 & 0.126 \\
QN2 (Leaf length) & 0.426 & -0.709 \\
QN1 (Leaf width) & 0.523 & -0.640 \\
QL3 (Stem color) & 0.909 & 0.323 \\
QL1 (Color of leaf surface) & 0.909 & 0.323 \\
\hline Cumulative variance (\%) & 20.4 & 14.5 \\
\hline
\end{tabular}




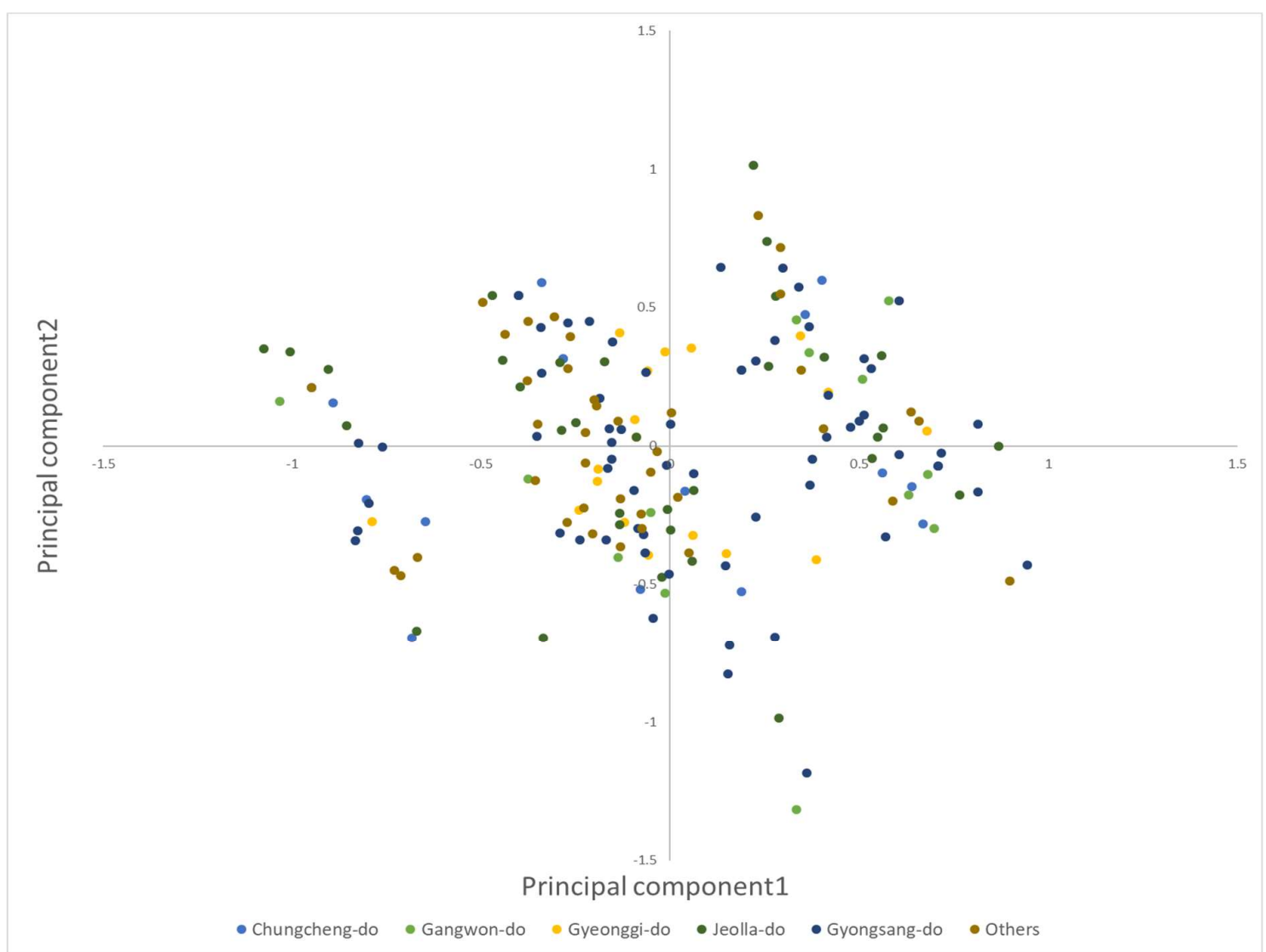

Figure 1. Projection of 183 accessions of cultivated $P$. frutescens var. frutescens of Perilla crop in the first and second principal components. $\circ$ : Gyeonggi-do, •: Gangwon-do, $\diamond$ : Chungcheong-do, $\diamond:$ Jeolla-do, $\square$ : Gyongsang-do, a: other region.

\subsection{SSR variation in accessions of cultivated P. frutescens var. frutescens of Perilla crop}

In total, 20 SSR loci were used to evaluate the genetic diversity and relationships among the 200 accessions of cultivated $P$. frutescens var. frutescens (Table 2). The 20 SSR primer sets confirmed a total of 137 alleles in the 200 accessions of cultivated $P$. frutescens var. frutescens. The number of alleles per locus ranged from 3 (KNUPF26, KNUPF77) to 13 (KNUPF10), and the average number of alleles per locus was 6.85 (Table 2). The average MAF was 0.470 , with a range of $0.225-0.835$. The average GD was 0.649 , with a range of $0.290-0.828$. The average PIC value was 0.599, with a range of 0.272-0.805 (Table 2). Among the 137 alleles, 31 private alleles (23\%) were only detected in one of the 200 accessions of cultivated $P$. frutescens var. frutescens. The percentage of rare alleles (frequency < 0.05 ) was 45\% (62 alleles) among the 137 alleles, whereas intermediate-frequency alleles (frequency of 0.05-0.5) and abundant alleles (frequency $>0.5)$ represented 50\% (68 alleles) and 5\% (7 alleles), respectively, of the total alleles (Supplement Fig. 2).

Table 2. Characteristics of the 20 SSR loci including allele size, number of allele, MAF, GD, PIC among 200 accessions of cultivated P. frutescens var. frutescens of Perilla crop.

\begin{tabular}{cccccc}
\hline SSR loci & Allele size & $\begin{array}{c}\text { No. of } \\
\text { Allele }\end{array}$ & MAF & GD & PIC \\
\hline KNUPF1 & $176-182$ & 5 & 0.835 & 0.290 & 0.272 \\
KNUPF2 & $156-166$ & 9 & 0.385 & 0.726 & 0.680 \\
KNUPF4 & $158-168$ & 7 & 0.370 & 0.708 & 0.654 \\
KNUPF5 & $177-183$ & 6 & 0.470 & 0.693 & 0.651 \\
KNUPF10 & $195-220$ & 13 & 0.565 & 0.648 & 0.626
\end{tabular}




\begin{tabular}{cccccc} 
KNUPF16 & $141-190$ & 13 & 0.225 & 0.828 & 0.805 \\
KNUPF23 & $204-214$ & 4 & 0.565 & 0.592 & 0.532 \\
KNUPF25 & $179-190$ & 5 & 0.485 & 0.629 & 0.561 \\
KNUPF26 & $153-264$ & 3 & 0.570 & 0.571 & 0.501 \\
KNUPF28 & $174-192$ & 6 & 0.660 & 0.522 & 0.484 \\
KNUPF33 & $186-213$ & 11 & 0.335 & 0.788 & 0.760 \\
KNUPF36 & $170-180$ & 6 & 0.390 & 0.749 & 0.712 \\
KNUPF37 & $256-259$ & 4 & 0.490 & 0.641 & 0.577 \\
KNUPF39 & $196-223$ & 9 & 0.410 & 0.711 & 0.662 \\
KNUPF43 & $127-155$ & 10 & 0.255 & 0.791 & 0.759 \\
KNUPF55 & $233-253$ & 11 & 0.520 & 0.681 & 0.653 \\
KNUPF59 & $152-170$ & 4 & 0.580 & 0.525 & 0.431 \\
KNUPF71 & $182-185$ & 4 & 0.435 & 0.645 & 0.572 \\
KNUPF74 & $190-198$ & 4 & 0.445 & 0.597 & 0.512 \\
KNUPF77 & $166-174$ & 3 & 0.405 & 0.651 & 0.576 \\
\hline Total & & 137 & & 0.649 & 0.599 \\
\hline Mean & & 6.85 & 0.470 & \\
\hline
\end{tabular}

MAF: major allele frequency, GD: gene diversity, PIC: polymorphic information content

To compare the geographical genetic variation of cultivated $P$. frutescens var. frutescens collected from five regions of South Korea and other region, this study also confirmed the number of alleles, MAF, GD, and PIC among the 200 accessions of cultivated P. frutescens var. frutescens (Table 3). The average number of alleles was 4 for the accessions of cultivated P. frutescens var. frutescens from the G1, G2, and G3 regions of South Korea and 5 for the G4 and G5 regions of South Korea and G6 region. The average MAF values were 0.537 for accessions of $\mathrm{G} 1$ region, 0.508 for accessions of $\mathrm{G} 2$ region, 0.555 for accessions of $\mathrm{G} 3$ region, 0.497 for accessions of G4 region, 0.500 for accessions of G5 region, and 0.601 for accessions of G6 region. The average GD values were 0.589 for accessions of G1 region, 0.594 for accessions of G2 region, 0.561 for accessions of G3 region, 0.623 for accessions of G4 region, 0.620 for accessions of G5 region, and 0.544 for accessions of G6 region. The average PIC values were 0.538 for accessions of G1 region, 0.530 for accessions of G2 region, 0.505 for accessions of G3 region, 0.570 for accessions of G4 region, 0.565 for accessions of G5 region, and 0.497 for accessions of G6 region (Table 3). From the results from among the six regions, the accessions of the G4 and G5 regions showed a relatively high genetic diversity value compared with those from the other regions of South Korea and other region, while the accessions of the G6 region showed the lowest genetic diversity value. 
Table 3. Estimates of MAF, allele number, gene diversity and PIC of 20 SSR loci in six regions collected from South Korea and other regions

\begin{tabular}{|c|c|c|c|c|c|c|c|c|c|c|c|c|c|c|c|c|c|c|c|c|c|c|c|c|}
\hline \multirow{2}{*}{ Marker } & \multicolumn{6}{|c|}{ No. of allele } & \multicolumn{5}{|c|}{ Major Allele Frequency } & \multicolumn{7}{|c|}{ Gene Diversity } & \multicolumn{6}{|c|}{$\mathrm{PIC}$} \\
\hline & G1 & $\mathrm{G} 2$ & G3 & $\mathrm{G} 4$ & G5 & G6 & $\mathrm{G} 1$ & $\mathrm{G} 2$ & G3 & G4 & G5 & G6 & G1 & G2 & G3 & G4 & G5 & G6 & G1 & $\mathrm{G} 2$ & G3 & G4 & G5 & G6 \\
\hline KNUPF1 & 2 & 2 & 2 & 4 & 3 & 4 & 0.947 & 0.923 & 0.900 & 0.889 & 0.851 & 0.667 & 0.100 & 0.142 & 0.180 & 0.205 & 0.265 & 0.491 & 0.095 & 0.132 & 0.164 & 0.198 & 0.249 & 0.433 \\
\hline KNUPF2 & 6 & 3 & 6 & 6 & 5 & 4 & 0.632 & 0.462 & 0.450 & 0.500 & 0.463 & 0.800 & 0.565 & 0.639 & 0.705 & 0.668 & 0.644 & 0.343 & 0.536 & 0.566 & 0.663 & 0.624 & 0.579 & 0.320 \\
\hline KNUPF5 & 5 & 4 & 3 & 4 & 6 & 5 & 0.737 & 0.692 & 0.500 & 0.694 & 0.507 & 0.444 & 0.438 & 0.485 & 0.605 & 0.485 & 0.661 & 0.688 & 0.416 & 0.451 & 0.527 & 0.452 & 0.616 & 0.638 \\
\hline KNUPF10 & 5 & 4 & 6 & 6 & 10 & 6 & 0.368 & 0.615 & 0.250 & 0.528 & 0.672 & 0.667 & 0.726 & 0.568 & 0.800 & 0.651 & 0.534 & 0.528 & 0.680 & 0.526 & 0.770 & 0.610 & 0.520 & 0.504 \\
\hline KNUPF16 & 5 & 5 & 5 & 8 & 11 & 7 & 0.316 & 0.385 & 0.400 & 0.250 & 0.284 & 0.689 & 0.765 & 0.722 & 0.715 & 0.823 & 0.811 & 0.505 & 0.726 & 0.676 & 0.668 & 0.799 & 0.785 & 0.485 \\
\hline KNUPF25 & 4 & 3 & 3 & 4 & 4 & 4 & 0.526 & 0.385 & 0.700 & 0.444 & 0.448 & 0.667 & 0.632 & 0.651 & 0.445 & 0.650 & 0.619 & 0.516 & 0.578 & 0.576 & 0.381 & 0.583 & 0.540 & 0.479 \\
\hline KNUPF26 & 3 & 3 & 3 & 3 & 3 & 3 & 0.474 & 0.462 & 0.750 & 0.444 & 0.537 & 0.711 & 0.637 & 0.615 & 0.395 & 0.634 & 0.591 & 0.440 & 0.565 & 0.535 & 0.347 & 0.558 & 0.517 & 0.386 \\
\hline KNUPF28 & 4 & 4 & 3 & 4 & 6 & 5 & 0.684 & 0.462 & 0.800 & 0.611 & 0.642 & 0.711 & 0.493 & 0.627 & 0.335 & 0.560 & 0.532 & 0.443 & 0.456 & 0.556 & 0.303 & 0.508 & 0.486 & 0.392 \\
\hline KNUPF33 & 6 & 4 & 7 & 7 & 7 & 7 & 0.421 & 0.538 & 0.250 & 0.472 & 0.343 & 0.356 & 0.704 & 0.627 & 0.820 & 0.715 & 0.778 & 0.784 & 0.657 & 0.576 & 0.795 & 0.684 & 0.747 & 0.757 \\
\hline KNUPF36 & 4 & 4 & 4 & 5 & 6 & 5 & 0.526 & 0.462 & 0.550 & 0.333 & 0.358 & 0.489 & 0.626 & 0.686 & 0.625 & 0.776 & 0.761 & 0.683 & 0.568 & 0.637 & 0.578 & 0.742 & 0.725 & 0.643 \\
\hline KNUPF37 & 3 & 3 & 3 & 4 & 4 & 4 & 0.474 & 0.462 & 0.800 & 0.500 & 0.582 & 0.578 & 0.632 & 0.639 & 0.335 & 0.619 & 0.578 & 0.591 & 0.558 & 0.566 & 0.303 & 0.549 & 0.519 & 0.537 \\
\hline KNUPF39 & 4 & 4 & 3 & 5 & 6 & 6 & 0.474 & 0.615 & 0.550 & 0.389 & 0.388 & 0.378 & 0.654 & 0.556 & 0.535 & 0.715 & 0.719 & 0.721 & 0.594 & 0.506 & 0.436 & 0.664 & 0.672 & 0.672 \\
\hline KNUPF59 & 3 & 3 & 3 & 3 & 4 & 2 & 0.632 & 0.538 & 0.650 & 0.694 & 0.537 & 0.511 & 0.521 & 0.556 & 0.485 & 0.440 & 0.558 & 0.500 & 0.455 & 0.465 & 0.406 & 0.365 & 0.468 & 0.375 \\
\hline KNUPF71 & 3 & 2 & 3 & 4 & 2 & 3 & 0.737 & 0.538 & 0.550 & 0.472 & 0.507 & 0.822 & 0.410 & 0.497 & 0.535 & 0.576 & 0.500 & 0.307 & 0.359 & 0.374 & 0.436 & 0.484 & 0.375 & 0.284 \\
\hline KNUPF74 & 2 & 3 & 3 & 3 & 3 & 4 & 0.632 & 0.538 & 0.600 & 0.444 & 0.507 & 0.489 & 0.465 & 0.556 & 0.560 & 0.610 & 0.589 & 0.595 & 0.357 & 0.465 & 0.499 & 0.527 & 0.506 & 0.513 \\
\hline KNUPF77 & 3 & 3 & 3 & 3 & 3 & 3 & 0.474 & 0.385 & 0.450 & 0.556 & 0.463 & 0.467 & 0.615 & 0.663 & 0.615 & 0.586 & 0.638 & 0.631 & 0.536 & 0.589 & 0.534 & 0.517 & 0.564 & 0.556 \\
\hline Averge & 4 & 4 & 4 & 5 & 5 & 5 & 0.537 & 0.508 & 0.555 & 0.497 & 0.500 & 0.601 & 0.589 & 0.594 & 0.561 & 0.623 & 0.620 & 0.544 & 0.538 & 0.530 & 0.505 & 0.570 & 0.565 & 0.497 \\
\hline
\end{tabular}

G1: Gyeonggi-do (19 accessions), G2: Gangwon-do (13 accessions), G3: Chungcheong-do (20 accessions), G4: Jeolla-do (36 accessions), G5: Gyongsang-do (67

accessions), G6: other region (45 accessions) 
2.4. Population structure and genetic relationships among accessions of var. frutescens from five regions of South Korea and other region

In the population structure among the 200 accessions of cultivated P. frutescens var. frutescens from the five regions of South Korea and other region, it was found that the highest value of $\Delta K$ for the 200 accessions of cultivated $P$. frutescens var. frutescens was for $K=3$ (Fig. 2). From the results, all accessions were divided into 3 main groups and an admixed group at $K=3$ as follows: Group I included 47 accessions from the G1 (one accession), G2 (7 accessions), G3 (2 accessions), G4 (14 accessions), and G5 (22 accessions) regions of South Korea and G6 (one accession) region. Group II comprised 37 accessions from the G3 (one accession) and G5 (2 accessions) regions of South Korea and G6 (34 accessions) region. Group III included 86 accessions from the G1 (16 accessions), G2 (4 accessions), G3 (14 accessions), G4 (19 accessions), and G5 (31 accessions) regions of South Korea and G6 (2 accessions) region. The admixed group included a total of 28 accessions, which came from the G2 (2 accessions), G3 (3 accessions), G4 (3 accessions), and G5 (12 accessions) regions of South Korea and G6 (8 accessions) other region (Fig. 3).

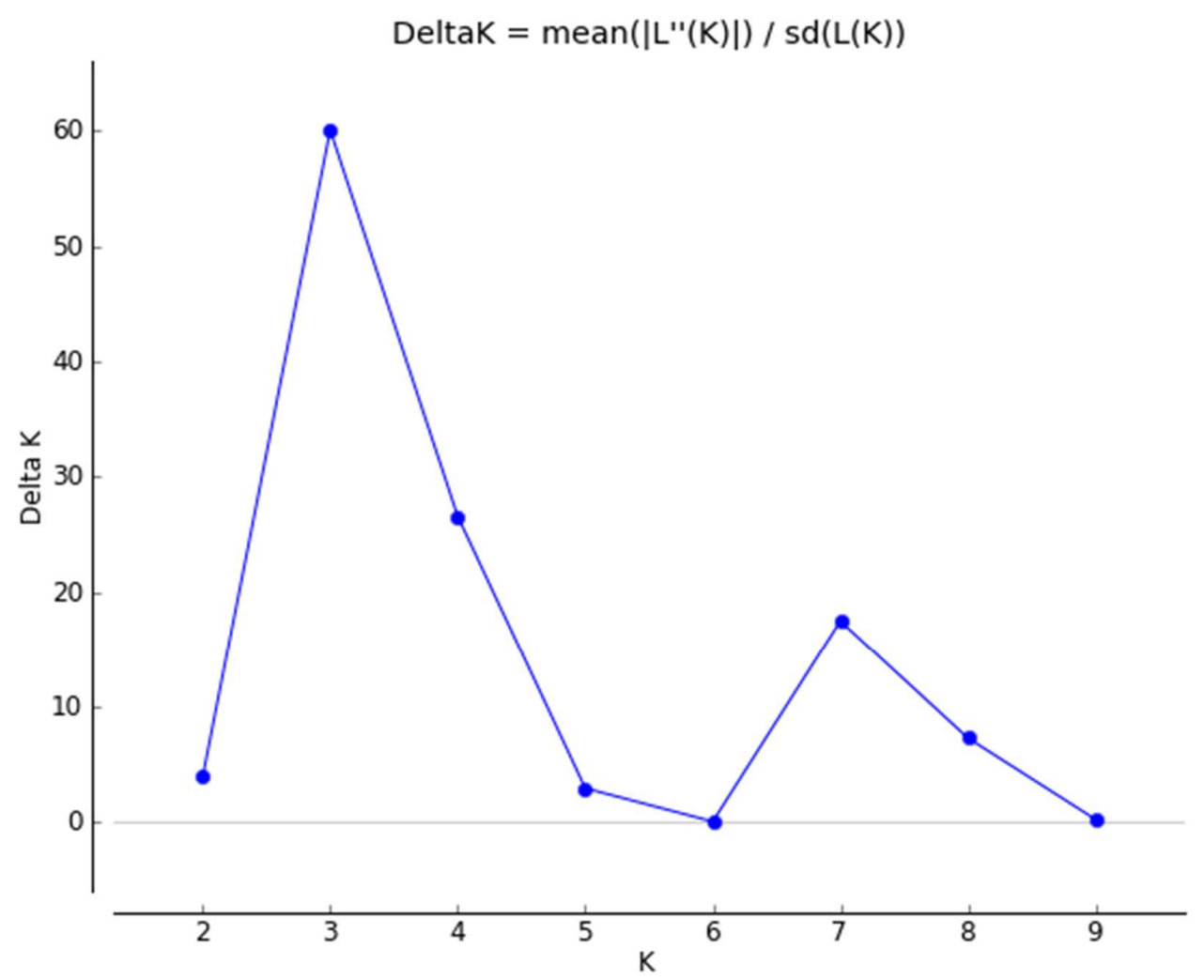

Figure 2. Magnitude of $\Delta \mathrm{K}$ as a function of $\mathrm{K}$. The peak value of $\Delta \mathrm{K}$ was at $K=3$, suggesting the existence of three main groups and an admixed group in the 200 accessions of cultivated $P$. frutescens var. frutescens of Perilla crop. 


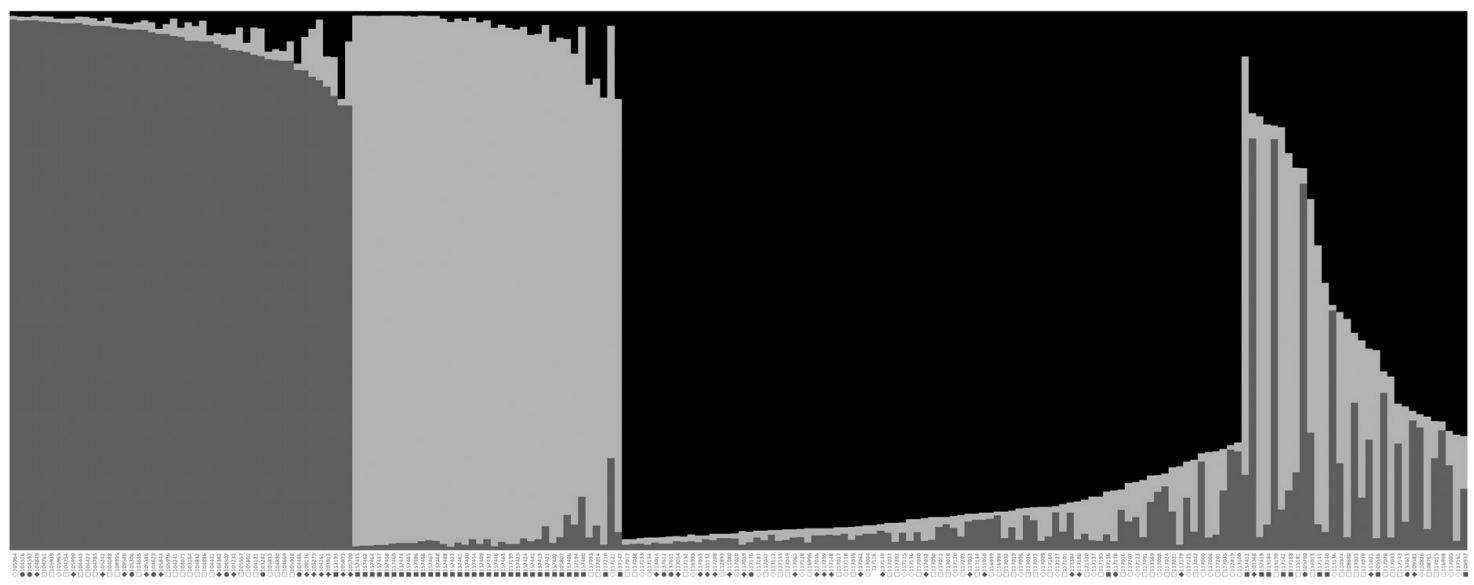

Figure 3. Population structure pattern for the highest $\Delta K$ value $(K=3)$ of 200 accessions of cultivated $P$. frutescens var. frutescens of Perilla crop. ○: Gyeonggi-do, •: Gangwon-do, $\diamond:$ Chungcheong-do, $\diamond:$ Jeollado, $\square$ : Gyongsang-do, $\mathbf{m}$ : other region.

On the other hand, in the phylogenetic tree constructed using UPGMA, the 200 accessions of cultivated $P$. frutescens var. frutescens were found to cluster into three major groups and an outgroup with a genetic similarity of $42 \%$ (Fig. 4). For the three major groups, Group I contained 145 accessions from five regions of South Korea and other region; Group II contained 19 accessions, which consisted of one accession from G1 region, two accessions from G2 region, eight accessions from G4 region, and eight accessions from G5 region; and Group III included four accessions, which consisted of two accessions from G3 region and two accessions from G4 region. The outgroup contained 32 accessions, which consisted of one accession from G3 region, four accessions from G4 region, seven accessions from G5 region, and 20 accessions from G6 region.

Furthermore, the accessions (145) of Group I were further subdivided into three groups with $45 \%$ genetic similarity as follows: Group I-1 contained 82 accessions and consisted of eight accession from G1 region, five accessions from $\mathrm{G} 2$ region, 11 accessions from G3 region, seven accessions from G4 region, 27 accessions from G5 region, and 24 accession from G6 region. Group I-2 contained 52 accessions and consisted of six accession from G1 region, six accessions from G2 region, five accessions from G3 region, 14 accessions from G4 region, and 21 accessions from G5 region. Group I-3 contained 11 accessions and consisted of two accession from G1 region, two accessions from G3 region, five accessions from G5 region, one accession from G4 region, and one accession from G5 region. The outgroup contained 32 accessions and divided into many sub groups as follows: three regions (one accession for G1, four accessions for G4, seven accessions for G5) of South Korea and other region ( 20 accessions for G6). As the results show, although some of the accessions collected in South Korea were included in the same group in accordance with geographic distribution, most accessions tended to be grouped differently from their collection areas. Meanwhile, the 45 accessions collected from other region were divided into two groups in the phylogenetic tree (Fig. 4). That is, some accessions belonged to the same group as the accessions collected in the five regions of South Korea, and the rest of the accessions were included in the outgroup along with some accessions collected in the five regions of South Korea. According to these results, the clustering patterns observed in this study were not clearly distinguished between and within the accessions of cultivated $P$. frutescens var. frutescens from the five regions of South Korea and the other region. 


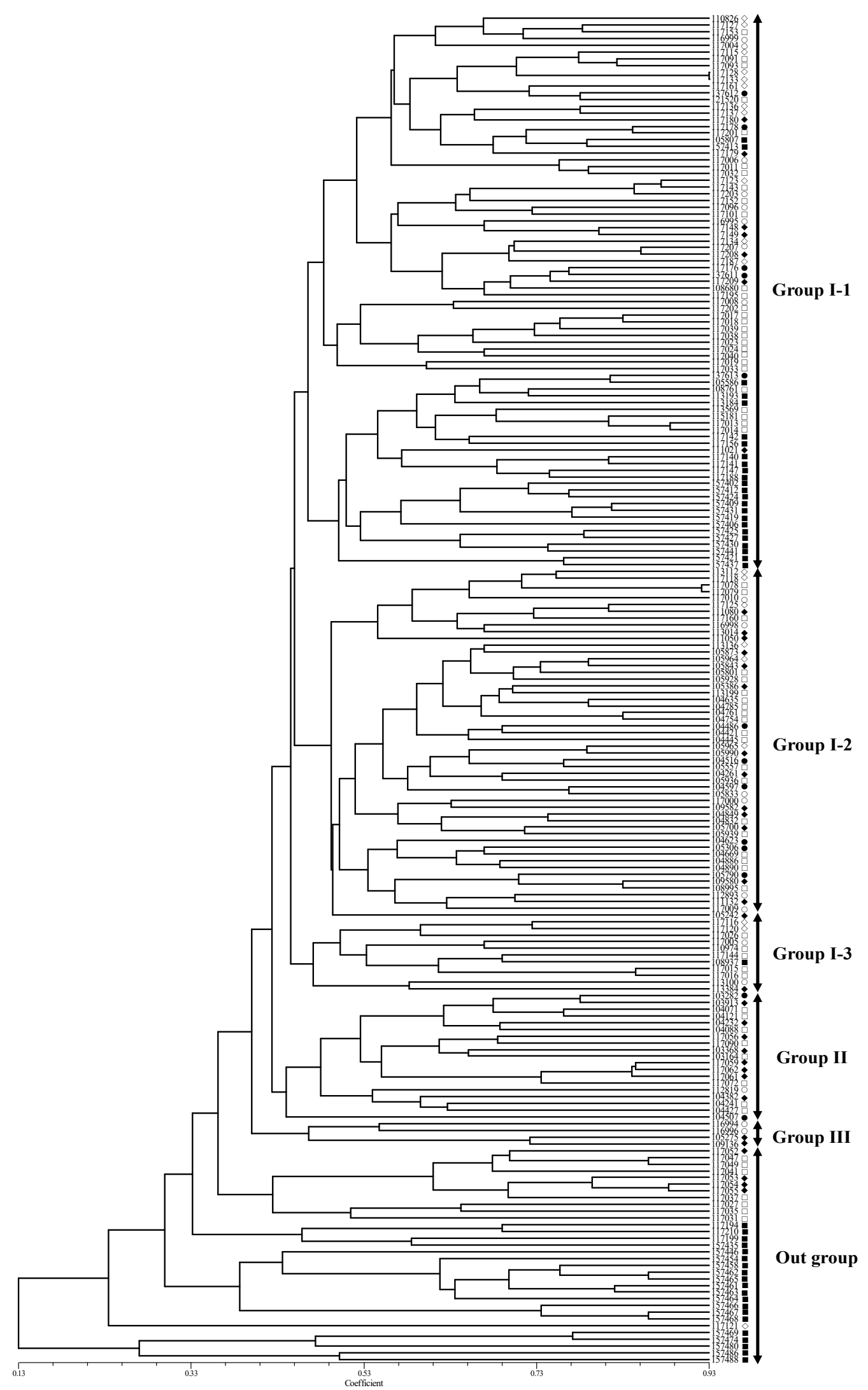

Figure 4. UPGMA dendrogram of 200 accessions of cultivated $P$. frutescens var. frutescens based on 20 SSR 
markers. The solid line represents the 11 major groups. ○: Gyeonggi-do, •: Gangwon-do, $\diamond$ : Chungcheong-do, $\bullet:$ Jeolla-do, $\square$ : Gyongsang-do, a: other region

\section{Discussion}

3.1. Morphological variations of cultivated P. frutescens var. frutescens in South Korea

Morphological variations of crop species within a geographic distribution and cultivation areas is of interest in the study of crop species evolution and differentiation [25-28]. In particular, domestication is a coevolutionary process that occurs when wild species are brought into cultivation by humans. This process arises as wild species are exposed to new selective environments associated with human cultivation and use that are essential for human survival $[29,30]$. Thus, cultivated crops from wild species are a direct product of human choice made over a long time $[24,26,27]$. This domestication process by humans for wild species has produced cultivated crops with unique features that make them suitable for agriculture [30, 31]. Therefore, the domestication of crops is a special case of crop evolution in which crop species adapt to human control and breed in human-controlled environments to support human survival.

As explained in the Introduction, the leaves and seed oil of cultivated $P$. frutescens var. frutescens have recently been in the limelight as health foods in South Korea because of the increased consumption of meat and the development of various cooking and usage methods for Perilla fresh leaves and seeds [9, 13, 14]. Therefore, the RDA-Genebank of South Korea is building a core population of genetic resources that can develop leaf and seed varieties among the preserved Perilla germplasm accessions. For the study of morphological variation of cultivated $P$. frutescens var. frutescens, we surveyed the morphological characteristics among 200 accessions of cultivated $P$. frutescens var. frutescens that were preserved in RDAGenebank and collected from South Korea.

In this study, 183 accessions of cultivated $P$. frutescens var. frutescens were examined for 8 qualitative and 3 quantitative characteristics. It was found that most accessions of cultivated $P$. frutescens var. frutescens were morphologically different in most of the morphological traits studied (Supplement Table 1). Among the morphological traits investigated in our study, the accessions of cultivated $P$. frutescens var. frutescens showed high frequency in the following traits: green for leaf and stem color, brown and dark brown for seed color, lanceolate and heart for leaf shape, slightly pubescent and normal pubescent for degree of pubescence, soft seed for seed hardness, a specific plant fragrance of cultivated $P$. frutescens var. frutescens for plant fragrance, a size of $8.1 \sim 10.0 \mathrm{~cm}$ for leaf width, a size of $12.1 \sim 14.0 \mathrm{~cm}$ for leaf length, and intermediate maturing type for flowering time. According to our results, most accessions of cultivated $P$. frutescens var. frutescens showed slight differences in the 8 qualitative and 3 quantitative traits (Supplement Table 1).

These results indicate that the accessions of cultivated $P$. frutescens var. frutescens exhibiting highest frequency characteristics for each morphological trait are related to their use as leaves or seeds in South Korea. For example, in South Korea $P$. frutescens var. frutescens is used similarly to vegetables such as lettuce; therefore, it is thought that many accessions showing green leaves and stems are favored and cultivated by farmers. In addition, the seeds of $P$. frutescens var. frutescens are used as oil or seasoning like sesame seeds; therefore, it is thought that many accessions with seeds showing soft and brown or dark brown colors are favored and cultivated by farmers. Lee et al. (1991) [10] reported that accessions of cultivated P. frutescens var. frutescens with brown or dark brown seeds were known to have a higher seed oil yield than those with white or gray seeds, while those with white seeds were known to have a higher protein content than those with black seeds. Furthermore, according to a report of field surveys by Lee et al. (2007) [32], accessions of cultivated $P$. frutescens var. frutescens showing brown and dark brown seed types were found more frequently than those showing white or gray seed types, and also they were widely distributed from northern to southern areas of South Korea. Meanwhile, white seed type accessions were found mainly in the southern part of South Korea. In interviews with farmers in the southern region of South Korea, Perilla seeds were found to be mainly used for seasoning and sesame seeds were used for cooking oil [32]. Therefore, in order to use seed oil or leafy vegetable varieties in South Korea, farmers are thought to have selected accessions of cultivated P. frutescens var. frutescens in accordance with their use in each region of South Korea.

According to the PCA results in our study, QL1, QL2, QL3, QL6, OL8, and QN1 contributed relatively significantly in the positive or negative direction on the first axis. In particular, most accessions of cultivated P. frutescens var. frutescens were separated on the positive and negative sides of the first axis (Fig. 1). Thus, these traits (QL1, QL2, QL3, QL6, OL8, QN1) that contributed in the positive or negative direction on the first or second axis may be thought of as being useful characteristics for discrimination between accessions of cultivated $P$. frutescens var. frutescens (Table 1). Therefore, the results of PCA in this study are expected to provide useful information for selecting accessions for developing seed oil or leafy vegetable varieties of 
cultivated $P$. frutescens var. frutescens in South Korea.

3.2. Genetic diversity and population structure of cultivated P. frutescens var. frutescens

Information on the genetic diversity, genetic relationships, and population structure of accessions of cultivated $P$. frutescens var. frutescens is an important basis for improvement of Perilla crop in South Korea. In particular, to maximize the use of genetic resources preserved in the RDA-Genebank of South Korea in breeding research, the genetic diversity and relationships among accessions of cultivated P. frutescens var. frutescens must be studied. In this study, we used 20 SSR markers for genetic diversity, genetic relationships, and population structure among accessions of cultivated $P$. frutescens var. frutescens. As explained in the Introduction, SSR markers have many advantages over the other molecular marker systems: simple experimental methods, high reproducibility, polymorphic genetic information contents, the codominant nature of SSR polymorphisms, and their abundance and distribution in genomes [19, 21, 22]. These advantages make them a suitable tool for studying genetic diversity, genetic relationships, and population structure in Perilla crop [9, 14, $20,23,33,34]$. In our study, we detected a total of 137 alleles with 20 SSRs segregating in the 200 accessions of cultivated $P$. frutescens var. frutescens examined, with an average of 6.85 alleles per locus. This value of alleles is higher than the numbers of effective alleles per SSR locus found in other crops, such as the values of 3.6 in barley [35], 6.7 in wheat [36], 4.8 in soybean [37], and 4.8 in pigeonpea [38]. As a result, it is thought that the native accessions of cultivated $P$. frutescens var. frutescens in South Korea have maintained relatively high genetic variation, as well as showing high variations in morphological characteristics.

In our study we analyzed the number of alleles, MAF, GD, and PIC among 200 accessions of cultivated $P$. frutescens var. frutescens collected from the G1 region (19 accessions), G2 region (13 accessions), G3 region (20 accessions), G4 region (36 accessions), and G5 region (67 accessions) of South Korea and G6 region (45 accessions) to compare the geographical variation of cultivated P. frutescens var. frutescens among the five regions of South Korea and other region (Table 3). The average GD values were 0.589, 0.594, 0.561, 0.623, 0.620, and 0.544 , respectively, for the accessions of cultivated $P$. frutescens var. frutescens from the five regions of South Korea and other region. Also, the average PIC values were 0.538, 0.530, 0.505, 0.570, 0.565, and 0.497, respectively, for the accessions of cultivated P. frutescens var. frutescens from the five regions of South Korea and other region. Although there was a difference in the number of plant materials collected and analyzed in the five regions of South Korea and other region, the accessions of G4 region showed the highest genetic diversity value among the six regions, while the accessions of G6 region showed the lowest genetic diversity value. In addition, among the six regions, in particular the accessions collected in the southern regions of Jeolla-do (G4) and Gyeongsang-do (G5) showed higher genetic diversity compared with the other regions (Table 3). Therefore, the present evaluation of genetic diversity in the five regions of South Korea and other region of cultivated $P$. frutescens var. frutescens using 20 SSR markers will provide information for expanding our understanding of regional genetic diversity in cultivated $P$. frutescens var. frutescens in South Korea. It is also necessary to collect farmers' lines or landraces to prevent genetic erosion of cultivated P. frutescens var. frutescens in South Korea. Therefore, the information provided here will help to characterize the introduction of new plants into the germplasm collection for conserving the accessions of cultivated $P$. frutescens var. frutescens. Also, it will help in selecting useful resources for the development of leafy-vegetable and seed-oil varieties of cultivated $P$. frutescens var. frutescens for the RDA-Genebank of South Korea.

In addition, information on the genetic relationships and population structure of the collected genetic resources of cultivated $P$. frutescens var. frutescens is essential for the efficient management and use of those collected genetic resources at the RDA-Genebank of South Korea [14]. In this study, to understand the genetic relationships and population structure of the 200 accessions of cultivated $P$. frutescens var. frutescens from five regions of South Korea and other region, we used the following two statistical methods: a model-based approach with STRUCTURE software and UPGMA dendrogram by the application of SAHN-Clustering from NTSYSpc V2.1. Particularly, cluster analysis can be used as an important tool for grouping the genetic resources of crop species to select useful genetic resources for the development of cultivars. In our study, the STRUCTURE results revealed that the 200 accessions of cultivated $P$. frutescens var. frutescens could be divided into 3 main groups and an admixed group at $K=3$ (Figs. 2, 3). At $K=3$, Group I included 47 accessions from the five regions of South Korea and other region. Group II comprised 37 accessions from the G3 and G5 regions of South Korea and G6 region. Group III included 86 accessions from the five regions of South Korea and other region. The admixed group included 28 accessions from the G2, G3, G4, and G5 regions of South Korea and G6 region. In addition, the UPGMA dendrogram results showed that the 200 accessions of cultivated $P$. frutescens var. frutescens were divided into three major groups and an outgroup with genetic similarity of $42 \%$ (Fig. 4). According to the results of the UPGMA and STRUCTURE analysis, there was no clear geographic location among the 200 accessions of cultivated P. frutescens var. frutescens from the G1, G2, G3, G4, and G5 regions in South Korea and the G6 
region (Figs. 3, 4). These results indicate that, in South Korea, landrace seeds of cultivated P. frutescens var. frutescens may be frequently exchanged by farmers among the five regions in South Korea through various routes over a long period of time, as previously reported by Lee et al. (2002) [5], Sa et al. (2013) [9] and Oh et al. (2020) [14]. The 45 accessions of other region were divided into two groups as follows: some accessions belonged to the same group as the accessions collected in the five regions of South Korea, and the rest of the accessions were included in the outgroup (Fig. 4). There is no clear genetic resource collection information for these accessions of other region, but these accessions of the outgroup can probably be considered foreign-introduced resources. Therefore, the results of the UPGMA and STRUCTURE analysis are expected to be helpful in understanding seed diffusion of native accessions of the cultivated $P$. frutescens var. frutescens in accordance with geographical distribution in South Korea.

In this study, genetic variation analysis was performed using morphological characteristics and SSR markers for accessions of cultivated P. frutescens var. frutescens collected in South Korea. In future, the results of this study are expected to provide useful information for the conservation of these genetic resources and the selection of useful resources for the development of varieties for seeds and leafy vegetables from cultivated $P$. frutescens var. frutescens in South Korea.

\section{Materials and methods}

\subsection{Plant materials}

For this study, 200 accessions of cultivated P. frutescens var. frutescens collected in South Korea and others (unknown accessions) were obtained from the RDA-Genebank of the Republic of Korea (http://genebank.rda.go.kr/, accessed on 1 November 2019). The accession numbers and location information for 200 Perilla accessions are shown in Supplement Table 2. In addition, Supplement Fig. 3 shows the collection areas of accessions of cultivated $P$. frutescens var. frutescens collected from South Korea, namely Gyeonggi-do (G1, 19 accessions), Gangwon-do (G2, 13 accessions), Chungcheong-do (G3, 20 accessions), Jeolla-do (G4, 36 accessions), and Gyeongsang-do (G5, 67 accessions). Meanwhile, 45 accessions were classified into other region (G6) because there was no information on where they were collected (Supplement Table 2). These accessions of cultivated $P$. frutescens var. frutescens were selected as genetic resources to be used as breeding materials for the development of leafy vegetable cultivars of Perilla crop from the Korean RDA-Genebank.

\subsection{Morphological characteristics analyzed}

To assess the morphological variation of the 200 accessions of cultivated P. frutescens var. frutescens, seven individuals of each accession were grown in a field at the experimental farm of Kangwon National University, Chuncheon, Gangwon-do. Approximately 20 seeds of each accession were sown in a nursery bed in early May and kept in a glass house for a month. Seven seedlings of each accession were then transplanted into the field in early June 2020. We examined three quantitative and eight qualitative characteristics, which were selected based on a previous report by Lee and Ohnishi (2001) [2], at the appropriate growth stages, as shown in Table 4. Measurements of the three quantitative characteristics, namely leaf width (QN1), leaf length (QN2), and flowering time (QN3), and observation of the 8 qualitative characteristics, namely color of leaf surface (QL1), color of reverse side leaf (QL2), stem color (QL3), seed color (QL4), leaf shape (QL5), degree of pubescence (QL6), seed hardness (QL7), and plant fragrance (QL8), were made on five individuals for each accession. However, 17 accessions in our study were not used for measurement because of plant growth failure in the field. Therefore, we conducted a morphological characteristics survey of only 183 accessions out of the 200 accessions of cultivated $P$. frutescens var. frutescens (Supplement Table 2). 
Table 4. Characters used in the morphological analysis of the 183 accessions of cultivated P. frutescens var. frutescens of Perilla crop

\begin{tabular}{|c|c|c|}
\hline \multicolumn{2}{|c|}{ Morphological character } & \multirow{2}{*}{$\begin{array}{l}\text { Category } \\
\text { 1-light green, 2-green, 3-deep green }\end{array}$} \\
\hline QL1 & Color of leaf surface & \\
\hline QL2 & Color of reverse side leaf & 1-light green, 2-green, 3-deep green \\
\hline QL3 & Stem color & 1-light green, 2-green, 3-deep green \\
\hline QL4 & Seed color & 1-white, 2-gray, 3-brown, 4-dark brown \\
\hline QL5 & Leaf shape & 1-lanceolate, 2-heart shape, 3-oblong \\
\hline QL6 & Degree of pubescence & 1-slightly pubescent, 2 -normal pubescent, 3 -heavily pubescent \\
\hline QL7 & Seed hardness & 1-soft, 2-hard \\
\hline QL8 & Plant fragrance & 1- plant fragrance of var. frutescens, 2-other plant fragrance \\
\hline QN1 & Leaf width & $1-8.0 \mathrm{~cm} \leq, 2-8.1 \sim 9.0 \mathrm{~cm}, 3-9.1 \sim 10.0 \mathrm{~cm}, 4-10.1 \sim 11.0 \mathrm{~cm}, 5-11.1 \sim 12.0 \mathrm{~cm}, 6-12.1 \sim 13.0 \mathrm{~cm}, 7-13.1 \mathrm{~cm}>$ \\
\hline QN2 & Leaf length & $1-10.0 \mathrm{~cm} \leq, 2-10.1 \sim 11.0 \mathrm{~cm}, 3-11.1 \sim 12.0 \mathrm{~cm}, 4-12.1 \sim 13.0 \mathrm{~cm}, 5-13.1 \sim 14.0 \mathrm{~cm}, 6-14.1 \sim 15.0 \mathrm{~cm}, 7-15.1 \mathrm{~cm}>$ \\
\hline QN3 & Flowering time & $\begin{array}{l}\text { 1-early maturing type (flowering days before August } 15) \text {, 2-intermediate maturing type ((flowering days from August } \\
15 \text { to September 5), 3-late maturing type (flowering days after September } 6 \text { ) }\end{array}$ \\
\hline
\end{tabular}




\subsection{SSR analysis and DNA electrophoresis}

For SSR analysis, the total DNA of 200 Perilla accessions was extracted from young leaf tissue of individual representative plants of each accession according to Plant DNAzol Reagent protocols (GibcoBRL Inc., Grand Island, NY, USA). In a preliminary test with over 100 SSR primer sets developed by many researchers $[18,19$, $23,39,40]$, we selected 20 SSR primer sets that showed high allele band amplification and a clear banding pattern in Perilla accessions (Supplement Table 3). The SSR amplification method for the Perilla crop has been described in a previous study by Kim et al. (2021) [23]. After polymerase chain reaction (PCR) amplification using Perilla SSR primer sets, DNA electrophoresis analysis was performed with a QIAxcel advanced system (QIAGEN Co., Hilden, Germany) according to the protocol described in the QIAxcel DNA Handbook. The samples were run in the QIAxcel advanced electrophoresis system, and sample separation was performed over $15 \mathrm{~min}$. Gel images were obtained as the results, and the quantification analysis was performed with QIAxcel software. The results were displayed as gel images and electropherograms acquired from the QIAxcel advanced system software.

\subsection{Data analysis}

Principal component analysis (PCA) was performed to detect differences between and within accessions of cultivated var. frutescens of Perilla crop. The PCA analysis was performed using the Microsoft Excel Statistical Analysis System Program and NTSYS-pc V2.1 Program [41]. SPSS software was used to perform correlation analysis for three quantitative and eight qualitative characteristics. Meanwhile, the fragments amplified for each SSR primer set were scored as present (1) or absent (0). Power Marker version 3.25 [42] was applied to obtain information on the number of alleles, allele frequency, major allele frequency (MAF), gene diversity (GD), and polymorphic information content (PIC). Genetic similarities (GS) were calculated for each pair of accessions using the Dice similarity index [43]. To illustrate the genetic relationships of the total accessions, a similarity matrix was used to construct an unweighted pair group method with arithmetic mean (UPGMA) dendrogram by the application of SAHN-Clustering from NTSYS-pc V2.1 [41]. Population structure was investigated for the 200 accessions of cultivated $P$. frutescens var. frutescens using STRUCTURE 2.2 software [44]. Five independent runs with $K$ values ranging from one to ten were performed with 100,000 cycles for both burn-in and run length. The delta $K$ statistic, based on the rate of change in the log probability of data between $K$ values [45], was calculated with STRUCTURE HARVESTER (http://taylor0.biology.ucla.edu/structHarvester/, accessed on 1 November 2020) based on the STRUCTURE results.

\section{Conclusion}

In this study, we evaluated the genetic variation and population structure of 200 accessions of cultivated Perilla frutescens var. frutescens collected from five regions of South Korea and other region using SSR markers and morphological characteristics. Among the morphological traits investigated in our study, the accessions of cultivated $P$. frutescens var. frutescens showed high frequency in the following traits: green for leaf and stem color, brown and dark brown for seed color, lanceolate and heart for leaf shape, slightly pubescent and normal pubescent for degree of pubescence, soft seed for seed hardness, the specific plant fragrance of cultivated $P$. frutescens var. frutescens for plant fragrance, a size of $8.1 \sim 10.0 \mathrm{~cm}$ for leaf width, a size of $12.1 \sim 14.0 \mathrm{~cm}$ for leaf length, and intermediate maturing type for flowering time. These results indicate that the accessions of cultivated $P$. frutescens var. frutescens exhibiting highest frequency characteristics for each morphological trait are related to their use as leaves or seeds in South Korea. In the PCA analysis, particularly color of leaf surface (QL1), color of reverse side leaf (QL2), stem color (QL3), degree of pubescence (QL6), plant fragrance (OL8), leaf width (QN1), and leaf length (QN2) contributed significantly in the positive or negative direction on the first axis. For analysis of SSR markers, the accessions of Jeolla-do (G4) and Gyeongsang-do (G5) regions showed a comparatively high genetic diversity value compared with those from other regions in South Korea. In the population structure and UPGMA analysis, there was no clear geographic structure among the 200 accessions of cultivated $P$. frutescens var. frutescens from the five regions of South Korea and other region. These results indicate that, in South Korea, landrace seeds of cultivated P. frutescens var. frutescens may be frequently exchanged by farmers among the five regions in South Korea through various routes. The results of this study are expected to provide useful information for the conservation of these genetic resources and the selection of useful resources for the development of varieties for seeds and leafy vegetables of cultivated P. frutescens var. frutescens in South Korea.

Supplementary Materials: Figure S1: Morphological features of two cultivated types of Perilla crop grown for investigation of morphological characteristics in experimental field: (a) Perilla frutescens var. 
frutescens and (b) P. frutescens var. crispa. Figure S2: Histogram of allele frequencies in 200 accessions of cultivated $P$. frutescens var. frutescens based on 20 SSR markers. Figure S3: Collection areas of 200 accessions of cultivated type of $P$. frutescens var. frutescens collected from five regions of South Korea and other region. For the regions of South Korea, 155 accessions were collected from Gyeonggi-do (G1, 19 accessions), Gangwon-do (G2, 13 accessions), Chungcheong-do (G3, 20 accessions), Jeolla-do (G4, 36 accessions), and Gyeongsang-do (G5, 67 accessions). However, collection areas of 45 accessions (G6 region) are not shown because there was no information on where they were collected. Table S1: Morphological variations of 183 accessions of cultivated P. frutescens var. frutescens of Perilla crop for 11 qualitative and quantitative traits in five regions of South Korea and other region. Table S2: Collection sites of Perilla accessions in five regions of South Korea and other region. Table S3: Characteristics of the 20 Perilla SSR loci used in the study.

Author contributions: D.Y. Hyun, S. Lee, and J.H. Rhee provided material samples and helped to draft the manuscript. J.Y. Oh, H. Park, and K.J. Sa performed the experiment and analyzed the data. J.Y. Oh, K.J. Sa, and J.K. Lee wrote the manuscript and designed the experiments. All authors have read and agreed to the published version of the manuscript.

\section{Funding: None}

Institutional Review Board Statement: Not applicable.

Informed Consent Statement: Not applicable.

Data Availability Statement: Data is contained within the article or Supplementary Materials.

Acknowledgments: This study was supported by the Basic Science Research Program through the National Research Foundation of Korea (NRF) funded by the Ministry of Education, Science and Technology (\#2016R1D1A1B01006461), and the Cooperative Research Program for Agriculture Science and Technology Development (project nos. PJ014227032019 and PJ0142272019), Rural Development Administration, Republic of Korea.

Conflicts of interest: The authors declare that they have no conflict of interest.

\section{References}

1. Makino, T. Makino's New Illustrated Flora of Japan; Hokuryukan Co.: Tokyo, Japan, 1961. (In Japanese)

2. Lee, J.K.; Ohnishi, O. Geographical differentiation of morphological characters among Perilla crops and their weedy types in East Asia. Breed Sci. 2001, 51, 247-255.

3. Lee, J.K.; Ohnishi, O. Genetic relationships among cultivated types of Perilla Frutescens and their weedy types in East Asia revealed by AFLP markers. Genet. Resour. Crop Evol. 2003, 50, 65-74.

4. Nitta, M. Origin Perilla Crops and Their Weedy Type. Ph.D. Thesis, Kyoto University, Kyoto, Japan, 2001; p. 78.

5. Lee, J.K.; Nitta, M.; Kim, N.S.; Park, C.H.; Yoon, K.M.; Shin, Y.B.; Ohnishi, O. Genetic diversity of Perilla and related weedy types in Korea determined by AFLP analyses. Crop Sci. 2002, 42, 2161-2166.

6. Nitta, M.; Lee, J.K.; Ohnishi, O. Asian Perilla crops and their weedy forms: Their cultivation, utilization and genetic relationships. Econ. Bot. 2003, 57, 245-253.

7. Ma, S.J.; Lee, J.K. Morphological variation of two cultivated types of Perilla crop from different areas of China. Korean J. Hortic. Sci. Biotechnol. 2017, 35,510-522.

8. Li, H.L. The vegetables of ancient china. Econ. Bot. 1969, 23, 235-260.

9. Sa, K.J.; Choi, S.H.; Ueno, M.; Park, K.C.; Park, Y.J.; Ma, K.H.; Lee, J.K. Identification of genetic variations of cultivated and weedy types of Perilla species in Korea and Japan using morphological and SSR markers. Genes Genom. 2013, 35, 649-659.

10. Lee, J.I.; Bang, J.K.; Lee, B.H.; Kim, K.H. Quality improvement in Perilla. I. Varietal differences of oil content and fatty acid composition. Kor. J. Crop Sci. 1991, 36, 48-61.

11. Asif, M. Health effects of omega-3,6,9 fatty acids: Perilla frutescens is a good example of plant oils. Orient Pharm. Exp. Med. 2011, 11, 51-59.

12. Lee, J.; Lee, M.H.; Cho, E.J.; Lee, S. High-yield methods for purification of a-linolenic acid from Perilla frutescens var. japonica oil. Appl. Biol. Chem. 2016, 59, 89-94. 
13. Park, H.; Sa K.J.; Hyun, D.Y.; Lee, S.; Lee, J.K. Identifying SSR Markers Related to Seed Fatty Acid Content in Perilla Crop (Perilla frutescens L.). Plants 2021, 10, 1404.

14. Oh, J.K.; Sa, K.J.; Hyun, D.Y.; Cho, G.T.; Lee, J.K. Assessment of genetic diversity and population structure among a collection of Korean Perilla germplasms based on SSR markers. Genes Genom. 2020, 42, 1419-1430.

15. Wilkes, H.G. Germplasm preservation: objectives and need. Pages 13-41in L. Knutson and A. K. Stoner, eds., Diversity and germplasm preservation. Global imperatives. Kluwer Academic Press, The Netherlands, 1989.

16. Williams, J.T. Plant Genetic Resources: Some New Directions. Advances in Agronomy. 1991, 45, 61-91.

17. Nitta, M.; Ohnishi, O. Genetic relationships among two Perilla crops, shiso and egoma, and the weedy type revealed by RAPD markers. Jpn. J. Genet. 1999, 74, 43-48.

18. Park, Y.J.; Dixit, A.; Ma, K.H.; Lee, J.K.; Lee, M.H.; Chung, C.S.; Nitta, M.; Okuno, K.; Kim, T.S.; Cho, E.G.; et al. Evaluation of genetic diversity and relationships within an on-farm collection of Perilla frutescens (L.) Britt. using microsatellite markers. Genet. Resour. Crop Evol. 2008, 55, 523-535.

19. Sa, K.J.; Choi, I.K.; Park, K.C.; Lee, J.K. Genetic diversity and population structure among accessions of Perilla frutescens (L.) Britton in East Asia using new developed microsatellite markers. Genes Genom. 2018, 40, 1319-1329.

20. Ha, Y.J.; Sa, K.J.; Lee, J.K. Identifying SSR markers associated with seed characteristics in Perilla (Perilla frutescens L.). Physiol. Mol. Biol. Plants 2021, 27, 93-105.

21. Powell, W.; Morgante, M.; Andre, C.; Hanafey, M.; Vogelet, J.; Tingey, S.; Rafalski, A. The comparison of RFLP, RAPD, AFLP and SSR (microsatellite) markers for germplasm analysis. Mol. Breed. 1996, 2, 225238.

22. Park, Y.J.; Lee, J.K.; Kim, N.S. Simple sequence repeat polymorphisms (SSRPs) for evaluation of molecular diversity and germplasm classification of minor crops. Molecules 2009, 14, 4546-4569.

23. Kim, J.Y.; Sa, K.Y.; Ha, Y.J.; Lee, J.K. Genetic variation and association mapping in $\mathrm{F}_{2}$ population of Perilla crop (Perilla frutescens L.) using new developed Perilla SSR markers. Euphytica 2021, 217, 135.

24. Schwanitz, F. The origin of cultivated plants. Harvard University Press, Cambridge, MA. 1966.

25. Gould, S.J; Johnston, R.F. Geographic variation. Ann. Rev. Ecol. Syst. 1972, 3, 457-498.

26. Harlan, J.R. Origins and processes of domestication. In G. P. Chapman [ed.], Grass evolution and domestication, 159-175. Cambridge University Press, Cambridge. 1992.

27. Harlan, J.R.; de Wet, J.M.J.; Price, G. Comparative evolution of cereals. Evolution 1973, 27, 322-325.

28. Wyatt, R.; Antonovics, J. Butterfly weed re-revisited: Spatial and temporal patterns of leaf shape variation in Asclepias tuberosa. Evolution 1981, 35, 529-542.

29. Purugganan, M.D.; Fuller, D.Q. The nature of selection during plant domestication. Nature 2009, 457, 843-848.

30. Purugganan, M.D. Evolutionary Insights into the Nature of Plant Domestication. Current Biology 2019, 29, 14, R705-R714

31. Vigouroux, Y.; Barnaud, A.; Scarcelli, N.; Thuillet, A.C. Biodiversity, evolution and adaptation of cultivated crops. C. R. Biologies 2011, 334, 450-457.

32. Lee. J.K.; Kim, N.S.; Ohnishi, O. Perilla crop and related weedy types collected in Korea. Korean J. Breed. Sci. 2007, 39, 316-323.

33. Ma, S.J.; Sa, K.J.; Hong, T.K.; Lee, J.K. Genetic diversity and population structure analysis in Perilla crop and their weedy types from northern and southern areas of China based on simple sequence repeat (SSRs). Genes Genom. 2019, 41, 267-281.

34. Lim, S.E.; Sa, K.J.; Lee, J.K. Bulk segregant analysis identifies SSR markers associated with leaf-and seed related traits in Perilla crop (Perilla frutescens L.). Genes Genom. 2021, 43, 323-332.

35. Hamza, S.; Hamida, W.B.; Rebaï, A.; Harrabi, M. SSR-based genetic diversity assessment among tunisian winter barley and relationship with morphological traits. Euphytica 2004, 135, 107-118.

36. Peng, J.; Bai, Y.; Haley, S.; Lapitan, N. Microsatellite-based molecular diversity of bread wheat germplasm and association mapping of wheat resistance to the Russian wheat aphid. Genetica 2009, 135, 95-122.

37. Tantasawat, P.; Trongchun, J.; Prajongjai, T.; Jenweerawat, S.; Chaowiset, W. SSR analysis of soybean (Glycine $\max$ (L.) Merr.) genetic relationship and variety identification in Thailand. Aust. J. Crop Sci. 2011, 5, 283-290.

38. Kimaro, D.; Melis, R.; Sibiya, J; Shimelis, H.; Shayanowako, A. Analysis of Genetic Diversity and Population Structure of Pigeonpea [Cajanus cajan (L.) Millsp] Accessions Using SSR Markers. Plants 2020, $9,1643$. 
39. Kwon, S.J.; Lee, J.K.; Kim, N.S.; Yu, J.W.; Dixit, A.; Cho, E.G.; Park, Y.J. Isolation and characterization of SSR. markers in Perilla frutescens Britt. Mol. Eco. Notes 2005, 5, 454-456.

40. Sa, K.J.; Lim, S.E.; Choi, I.K.; Park, K.C.; Lee, J.K. Development and Characterization of New Microsatellite Markers for Perilla frutescens (L.) Britton. Amer. J. Plant Sci. 2019, 10, 1623-1630.

41. Rohlf, F. NTSYS-pc: Numerical Taxonomy and Multivariate Analysis System; Version Exter Software: Setauket, NY, USA, 2000.

42. Liu, K.; Muse, S.V. PowerMarker: An integrated analysis environment for genetic marker analysis. Bioinformatics 2005, 21, 2128-2129.

43. Dice, L.R. Measures of the amount of ecologic association between species. Ecology 1945, 26, $297-302$.

44. Pritchard, J.K.; Wen,W. Documentation for STRUCTURE Software: Version. 2003. Available online: http://www.pritc.h.bsd. uchicago.Edu (accessed on 10 December 2020)

45. Evanno, G.; Regnaut, S.; Goudet, J. Detecting the number of clusters of individuals using the software. STRUCTURE: A simulation study. Mol. Ecol. 2005, 14, 2611-2620. 